\title{
An Unusual Atrial Septal Defect Producing Symptoms in a Young Lady
}

\author{
Md. Mashiul Alam ${ }^{1}$, Md. Al-Amin ${ }^{2}$ \\ ${ }^{1}$ Assistant professor of cardiology, Jahurul Islam Medical College and Hospital (JIMCH), Kishoregonj, Bangladesh \\ ${ }^{2}$ Medical Officer, National Institute of Cardiovascular Disease (NICVD), Dhaka, Bangladesh
}

Corresponding author: Md. Mashiul Alam; mashiull@gmail.com

Received 06 January 2020;

Accepted 25 January 2020;

Published 04 February 2020

\begin{abstract}
Atrial septal defect is the most common congenital heart disease among adult population. Secundum variety of atrial septal defect comprises most frequently encountered atrial septal anomaly which presents during late age. Primum atrial septal defect usually associated with atrioventricular cushion defect among patients with Down syndrome. Small number of patients may present with fenestrated atrial septal defect. We have presented case of a woman who is young and symptomatic due to atrial septal defect comprising both secundum and primum type.
\end{abstract}

Keywords: Atrial Septal Defect, ASD primum, ASD secundum.

\section{Introduction}

Atria septal defect (ASD) is a common congenital heart disease among adults. ${ }^{[1]}$ It accounts for $22-40 \%$ of congenital heart disease in adults and $10 \%$ of all congenital heart disease. ${ }^{[2]}$ Atrial septal defect may occur in variable positions of inter-atrial septum. In $75 \%$ of cases this defect affect middle portion of inter-atrial septum or in the area of fossa ovalis. A small portion of such defect may manifest as multiple openings or fenestrated ASD. ${ }^{[3]}$ Lower portion of atrial septum is affected in around $15 \%$ of cases which is called ASD primum. ASD involving both middle \& lower segment of inter-atrial septum is a rare anomaly and under reported in literature. We have presented a case of 37-year-old young lady who presented with fatigue, shortness of breath \& palpitation where transthoracic echocardiography revealed combined secundum \& primum ASD. To the best of our knowledge such type of presentation is a rare finding and no such case has been reported in isolation apart from Down syndrome.

\section{Case report}

A 37-year-old young woman presented at out-patient department with shortness of breath, fatigue, palpitations for years which had been increasing for months. Her present complaint depicted dyspnea with NYHA class III heart failure which increased upon exertion with no seasonal variation. She was not previously diagnosed as a case of bronchial asthma. She was mother of one child who was born by normal vaginal delivery at home 5 years ago. Her pregnancy was uneventful and she had some shortness of breath during delivery of her child. On enquiry she had no childhood history of rheumatic fever but was little out of breath during play time with her peers. Her parents were alive and in good health. She has got 3 siblings and all are doing well.
Physical examination revealed mild dyspnea with decubitus on choice. Her vitals were normal. JVP was $4 \mathrm{~cm}$ above sternoclavicular joint. There was mild pitting edema.

Precordial examination showed left parasternal heave, no palpable $\mathrm{P} 2$ or thrill. On auscultation there was a grade $3 / 6$ ejection systolic murmur over pulmonary area which did not radiate to anywhere. Lung bases were clear to auscultate bilaterally.

Transthoracic echocardiogram revealed right atrium and ventricle were mildly dilated in comparison to left side of heart. Diameter of RV at the base during diastole was $48 \mathrm{~mm}$. There was two defects in the atrial septum best shown in apical 4 chamber view. One defect was at the middle of IAS with adequate rim with a diameter around $15 \mathrm{~mm}$. Another defect was present at the bottom of IAS with intact inter-ventricular septum and atrioventricular valves. Diameter of the latter defect was about $20 \mathrm{~mm}$. [Figure 1]

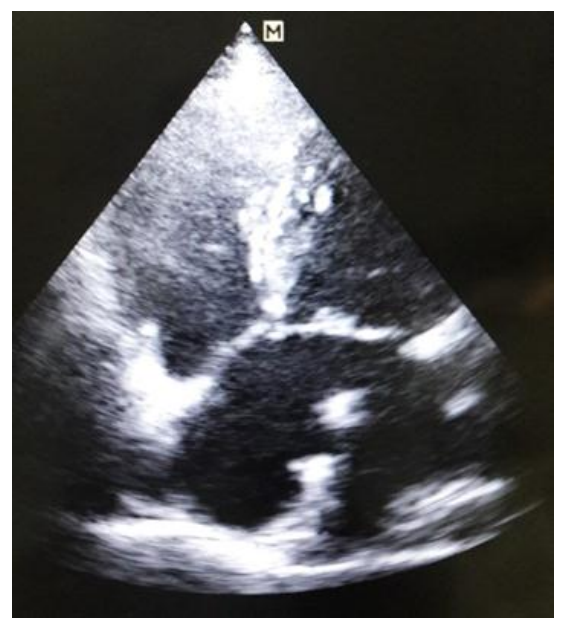

Figure 1: Apical 4 chamber view of 2D echocardiography showing ASD secundum and primum together. 
Colour Doppler echocardiogram showed two jets crossing from left atrium (LA) to right atrium (RA) through the above mentioned IAS defects towards the tricuspid valve. There was small TR jet visible during ventricular systole which was overlapped with flows from LA to RA. [Figure $2 \& 3$ ]

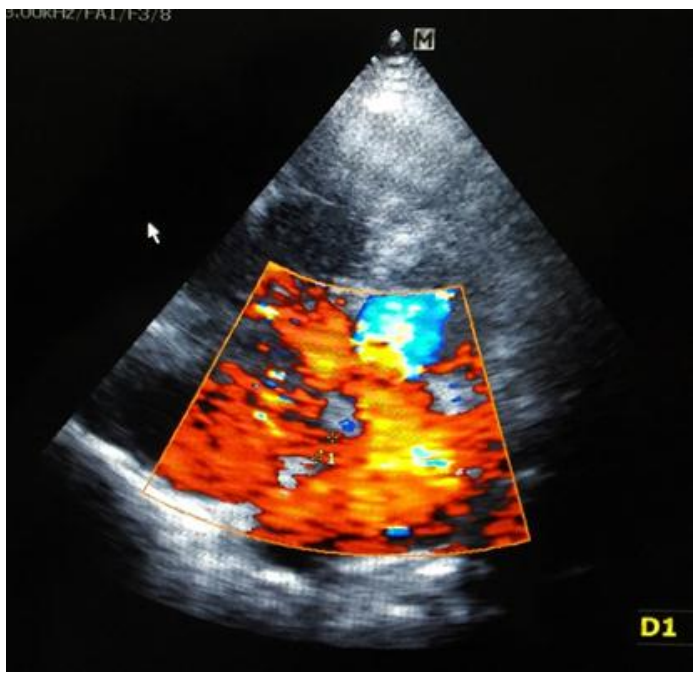

Figure 2: Color Doppler echo in apical 4 chamber view showing two laminar jets from LA to RA.

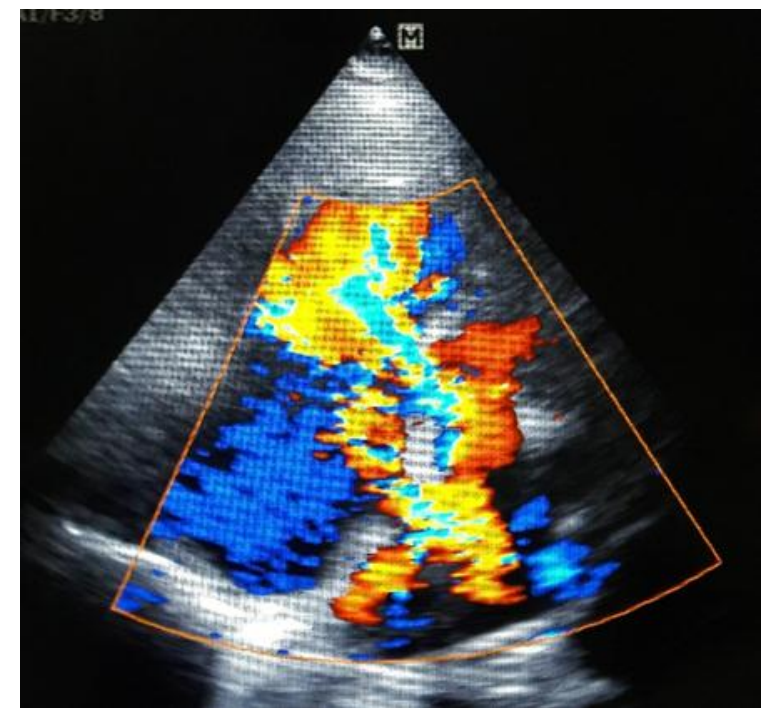

Figure 3: Color Doppler echo in sub-costal view showing ASD secundum and primum.

Apart from these findings there was no other echocardiographic abnormality. So the patient was diagnosed as having both secundum \& primum ASD. Patient was referred to higher cardiac center for further evaluation and management.

\section{Discussion}

Atrial septal defect is a common congenital heart disease where defect may occur at various position of the atrial septum. Secundum ASD is most common pattern followed by septum primum, sinus venosus type \& coronary sinus type ASD. ${ }^{[2]}$ Small portion of ASD may have multiple openings with in the septum. ${ }^{[3]}$ This fenestrated type ASD has openings with in the septum with some septal rim.

ASD may be associated with different other anomalies namely anomalous pulmonary venous return, ventricular septal defect, endocardial cushion defect, pulmonary stenosis etc. It is also acts as helpful shunt in different life threatening congenital heart disease, for example, transposition of great arteries, truncus arteriosus etc. But ASD secundum and primum together is underreported in articles.

Endocardial cushion defect which is often associated with Down syndrome in children has ASD primum associated with atrio-ventricular valve defect. It may have simultaneous opening in the middle of atrial septum along with ASD primum.

ASD patients are asymptomatic in their early life and symptoms develop with age. About $90 \%$ patient, who are untreated develops symptoms of exertional shortness of breath, palpitation, fatigue or arrhythmia by the age of 40 years. ${ }^{[4]}$

Echocardiography can establish the size \& location of ASD, the magnitude of shunt, hemodynamic changes due to the shunt, degree of pulmonary hypertension, other associated anomalies. ${ }^{[5]}$ Transthoracic echocardiography has higher sensitivity to detect ASD primum \& secundum in comparison to sinus venosus type ASD.

There are percutaneous and surgical methods for closure of atrial septal defect. Selection of time to close \& method of closure depends on patient's clinical symptoms, location of ASD, reversibility \& degree of pulmonary hypertension and presence of other associated anomalies. ${ }^{[6]}$

When the ASD is closed before the age of 25 years and pulmonary arterial systolic pressure (PASP) is less than $40 \mathrm{mmHg}$ long term prevention of death $\&$ disability is best achieved. ${ }^{[6]}$

\section{References}

[1] Lloyd TR, Rao PS, Beekman RH, Mendelsohn AM, Sideris EB: Atrial septal defect occlusion with the button device (a multi-institutional U.S. trial). Am J Cardiol. 1994; 73 (4):286-91.

[2] Diaconu CC. Atrial Septal Defect in an elderly woman- a case report. J Med Life. 2011 Feb 15; 4(1):91-93.

[3] Mitchell AR, Roberts P, Eichhofer J, Timperley J, Ormerod OJ. Echocardiographic assessment and percutaneous closure of multiple atrial septal defects. Cardiovasc Ultrasound 2004; 2:9.

[4] Saxena A, Divekar A, Soni NR. Natural history of secundum atrial septal defect revisited in the era of transcatheter closure. Indian Heart J. 2005;57:35-38.

[5] Valdes-Cruz LM, Cayre RO. Echocardiographic diagnosis of congenital heart disease. Philadelphia:1998.

[6] Berger F, Ewert P. Atrial septal defect: waiting for symptoms remains an unsolved medical anachronism. Eur Heart J. 2010. 